"This document is the Accepted Manuscript version of a Published Work that appeared in final form in Journal of the American Chemical Society copyright (C) American Chemical Society after peer review and technical editing by the publisher. To access the final edited and published work see [insert ACS Articles on Request authordirected link to Published Work, see http://pubs.acs.org/doi/abs/10.1021/jacs.5b03340

\title{
Ni-catalyzed Divergent Cyclization/Carboxylation of Unactivated Primary and Secondary Alkyl Halides with $\mathrm{CO}_{2}$
}

\author{
Xueqiang Wang ${ }^{\dagger \dagger}, \mathrm{Yu} \mathrm{Liu}^{\dagger \dagger}$ and Ruben Martin ${ }^{\dagger \S *}$ \\ $\dagger$ Institute of Chemical Research of Catalonia (ICIQ), Av. Països Catalans 16, 43007 Tarragona, Spain \\ $\S$ Catalan Institution for Research and Advanced Studies (ICREA), Passeig Lluïs Companys, 23, 08010, Barcelona, Spain
}

Supporting Information Placeholder

\begin{abstract}
A user-friendly Ni-catalyzed reductive cyclization/carboxylation of unactivated alkyl halides with $\mathrm{CO}_{2}$ is described. The protocol operates under mild conditions with excellent chemoselectivity profile and a divergent syn/anti-selectivity pattern that can be easily modulated by the substrate utilized.
\end{abstract}

Catalytic reductive coupling reactions of organic halides have evolved from mere curiosities to robust tools that rapidly build up molecular complexity from simple precursors. ${ }^{1}$ At present, this field of expertise remains essentially confined to bond-formation events at the initial site (Scheme 1, path a). Intriguingly, the ability to promote cascade reactions of unactivated alkyl electrophiles via multiple $\mathrm{C}-\mathrm{C}$ bond-formations has virtually been unexplored (path b). ${ }^{2,3}$ If successful, such protocols would offer a unique opportunity to increase our chemical portfolio for rapidly preparing carbocyclic skeletons while dealing with bond-formation events at distal sites.

\section{Scheme 1. Bond-Formation via Electrophile Couplings}

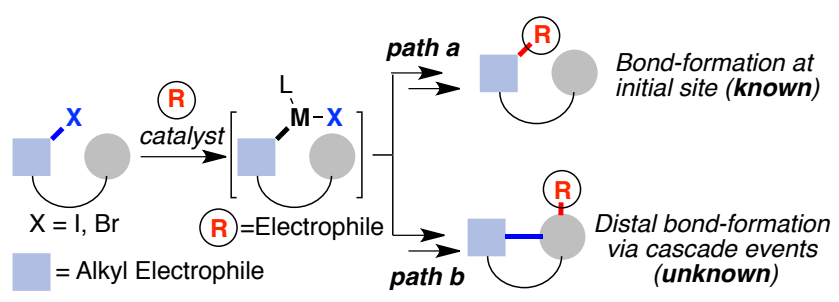

In recent years, $\mathrm{we}^{4}$ and others ${ }^{5}$ have designed new catalytic reductive carboxylation techniques of organic hal- ides using $\mathrm{CO}_{2}$, probably the greenest $\mathrm{C} 1$ synthon in nature. ${ }^{6}$ Unlike the utilization of stoichiometric amounts of organometallic complexes, ${ }^{7}$ many of these protocols operate under mild conditions and in the absence of sensitive reagents, thus representing a straightforward, yet practical, alternative for preparing carboxylic acids, privileged motifs in a myriad of pharmaceuticals. ${ }^{8}$ At the outset of our investigations, however, it was unclear whether $\mathrm{CO}_{2}$ could participate in cascade reductive coupling reactions via multiple bond-forming reactions. ${ }^{9}$ Although we anticipated that reductive cascade processes based on the employment of unactivated alkyl halides, ${ }^{10}$ probably the most challenging substrates in the cross-coupling arena, would be rather problematic, we were attracted to the challenge. ${ }^{11}$ Specifically, such a route would offer the unique opportunity to control parasitic $\beta$-hydride elimination pathways ${ }^{10}$ while resulting in carboxylated carbocyclic skeletons from simple precursors via distal catalytic $\mathrm{CO}_{2}$ fixation. We speculated that a technique capable of modulating, at will, the anti/synselectivity of the cyclization event would set the standards for catalytic biomimetic cascade carboxylation events. ${ }^{12}$ Herein, we report a mild and user-friendly reductive cyclization/carboxylation of unactivated alkyl halides with $\mathrm{CO}_{2}$ en route to elusive tetrasubstituted olefins (Scheme 2, path b) ${ }^{13}$ In sharp contrast with syncarbometallation techniques of stoichiometric, welldefined and, in many instances, air-sensitive organometallics (Scheme 2, path a) ${ }^{14}$ our protocol is characterized by its exquisite chemoselectivity profile while obviating the need for sensitive species. Importantly, this trans- 
formation is distinguished by an unconventional divergence in syn/anti-selectivity that can be easily dictated by the ligand backbone or substrate utilized.

Scheme 2. Cyclization/Functionalization of Alkyl Halides

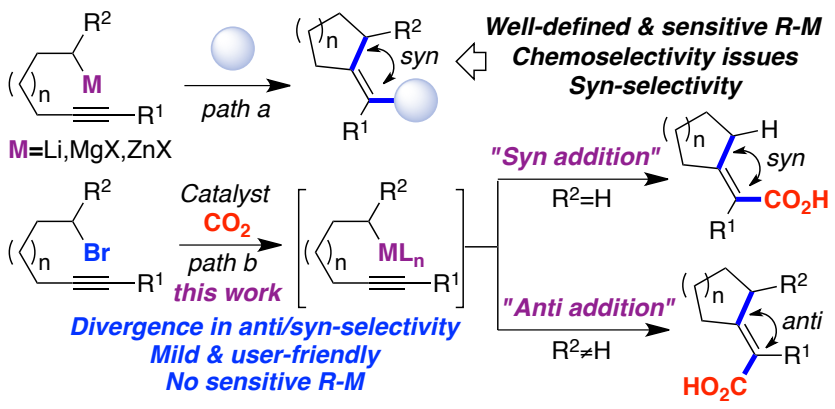

We began our investigations by studying the catalytic cascade cyclization/carboxylation reaction of 1 aa at atmospheric pressure of $\mathrm{CO}_{2}$ utilizing $\mathrm{NiCl}_{2} \cdot$ glyme as the catalyst and $\mathrm{Mn}$ as the reductant in DMF at rt (Table 1). ${ }^{15}$ As for related catalytic reductive coupling processes, ${ }^{1}$ we anticipated that subtle differences in the ligand backbone would exert a profound influence on the reaction outcome. As shown in Table 1, this turned out to be the case; while L1-L4 predominantly resulted in nonproductive $\beta$-hydride elimination pathways (entries 1-4), the inclusion of ortho-substituents in the phenanthroline backbone cleanly produced 2a. Among them, L5 and L6 allowed for obtaining $\mathbf{2 a}$ in a respectable $33 \%$ and $39 \%$ yield (entries 5 and 6) with no observable side products. Strikingly, the precatalyst (entries 7-9), solvent (entries 11-12) and reductant utilized (entry 10) had a nonnegligible effect on reactivity, suggesting an intimate interplay between all reaction parameters. While not anticipated, we found the best results with a priori less activated alkyl bromide 1a (entry 10 vs 8), giving rise to 2a in a $85 \%$ isolated yield. ${ }^{16}$ Importantly, not even traces of 3a were found in the crude reaction mixtures. In line with our expectations, control experiments revealed that all reaction parameters $\left(\mathrm{NiBr}_{2} \cdot\right.$ diglyme, $\mathbf{L 6}, \mathrm{Mn}$ and DMF) were critical for success.

Table 1. Optimization of the Reaction Conditions. ${ }^{a}$

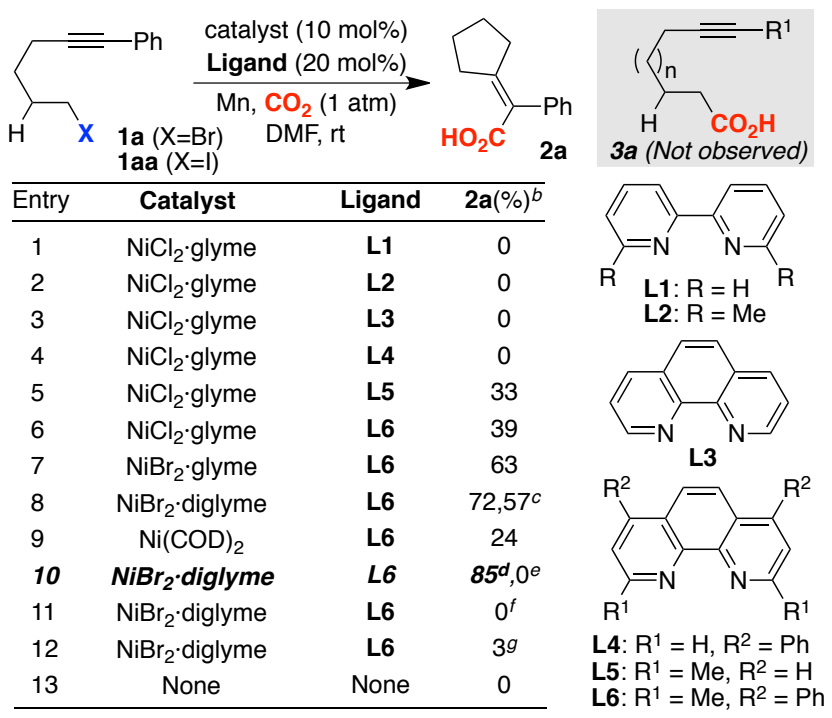

${ }^{a}$ 1aa $(0.30 \mathrm{mmol})$, Ni catalyst (10 mol\%), L (20 mol\%), Mn (2.20 equiv.), DMF $(0.15 \mathrm{M}), \mathrm{CO}_{2}(1 \mathrm{~atm})$ at $\mathrm{rt}$ overnight. ${ }^{b}$ Determined by HPLC using naphthalene as internal standard. ${ }^{c} \mathrm{NiBr}_{2} \cdot$ diglyme $(5 \mathrm{~mol} \%)$. ${ }^{d}$ Using 1a $(0.30$ $\mathrm{mmol}$ ); isolated yield, ${ }^{e} \mathrm{Without} \mathrm{Mn}$ or with $\mathrm{Zn}$ as reductant. ${ }^{f}$ DMA as solvent. ${ }^{g} \mathrm{MeCN}$ as solvent.

Encouraged by these results, we turned our attention to study the preparative scope of our catalytic cyclization/carboxylation reaction. Particularly noteworthy was the functional group tolerance of our protocol, as ketones $(\mathbf{2} \mathbf{d})$, ethers $(\mathbf{2} \mathbf{b}, \mathbf{2} \mathbf{i})$, esters $(\mathbf{2} \mathbf{e}, \mathbf{2} \mathbf{j})$, amides $(\mathbf{2} \mathbf{f})$, alkenes $(\mathbf{2 m})$ or heterocycles $(\mathbf{2 o}, \mathbf{2 p})$ were all perfectly accommodated. Undoubtedly, the exquisite chemoselectivity profile of our transformation represents a bonus when compared with classical carbometalation techniques based on the utilization of organolithium or Grignard reagents, among others (Scheme 2, path a). ${ }^{14}$ As shown for $\mathbf{2 g}$, the inclusion of ortho substituents on the aromatic motif did not hamper the reaction. Interestingly, we found that the cyclization/carboxylation event could be even conducted in the presence of electrophilic partners that are suited for Ni-catalyzed reductive carboxylation reactions such as aryl chlorides $(\mathbf{2 l}),{ }^{5 \mathrm{c}}$ tosylates $(\mathbf{2 k})$ or pivalates $(\mathbf{2 j}) ;{ }^{4 \mathrm{c}}$ notably, no traces of the corresponding benzoic acids derived from a $\mathrm{C}-\mathrm{Cl}$ or $\mathrm{C}-\mathrm{O}$ bondcleavage were detected in the crude reaction mixtures, thus providing ample opportunities for further functionalization. While one might argue that such protocol would essentially be restricted to five-membered rings or alkyne residues possessing aromatic motifs, the preparation of $\mathbf{2 n , ~} \mathbf{2 0}, \mathbf{2 p}$ or $\mathbf{2 q}$ clearly indicates otherwise. Strikingly, free alkynes posed no problems (2h); such finding is certainly remarkable taking into consideration the proclivity of terminal alkynes towards competitive trimerization pathways. ${ }^{17}$ As anticipated from a classical syn-carbometalation via in situ generated alkylnickel species, ${ }_{1}^{14,18}$ we obtained $\mathbf{2 r}$ and $\mathbf{2 s}$. The structure of $\mathbf{2 r}$ was univocally established by X-ray crystallographic analysis. ${ }^{15}$ 
Table 2. Scope Unactivated Primary Alkyl Bromides. ${ }^{a, b}$

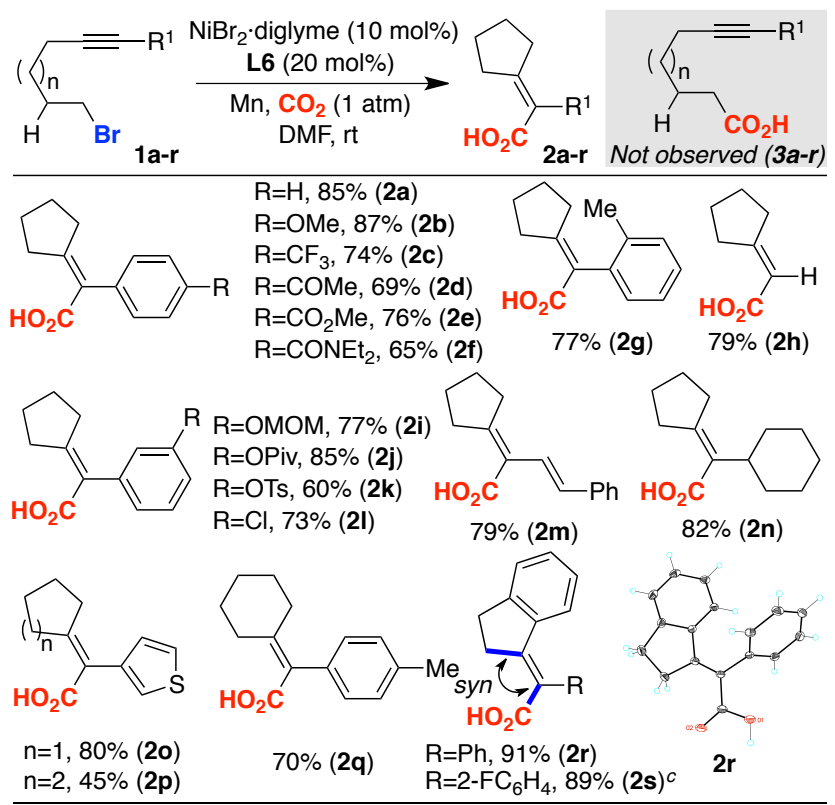

${ }^{a}$ As for Table 1 , entry $10 .{ }^{b}$ Isolated yields, average of at least two independent runs. ${ }^{c} E / Z=19: 1$.

Next, we focused our attention on a more challenging scenario dealing with unactivated secondary alkyl halides. ${ }^{10}$ These substrates are particularly problematic due to their reluctance to undergo oxidative addition and their propensity towards non-productive $\beta$-hydride elimination, thus constituting an opportunity to explore the robustness of our cyclization/carboxylation event (Table 3). Strikingly, the employment of $\mathbf{4 a}$ using $\mathbf{L 6}$ under otherwise similar reaction conditions to that of Table 2 resulted in an unexpected selectivity switch $\left(\mathbf{5 a}: 5 \mathbf{a}^{\prime}=3.3: 1\right)$. In a formal sense, $\mathbf{5 a}$ can be derived from a rather elusive anti-carbometalation event. ${ }^{19}$ Although 5a was fully characterized by NMR spectroscopical analysis, X-ray crystallography unambiguously identified the abnormal anti-selective motion. ${ }^{15}$ It is worth noting that the preparation of 5a represents the first reductive carboxylation that can be conducted with unactivated secondary alkyl electrophiles. Interestingly, the anti-selectivity could be modulated by the ligand employed. Specifically, we found that $\mathbf{L 5}$ uniquely afforded 5a with little amounts of $\mathbf{5 a}$ ' being present in the crude mixtures $\left(\mathbf{5 a}: \mathbf{5 a} \mathbf{a}^{\prime}=12.5: 1\right)$. At present, we have no rationale explanation for this intriguing behavior. ${ }^{20}$

Table 3. Scope Unactivated Secondary Alkyl Bromides. ${ }^{a, b}$

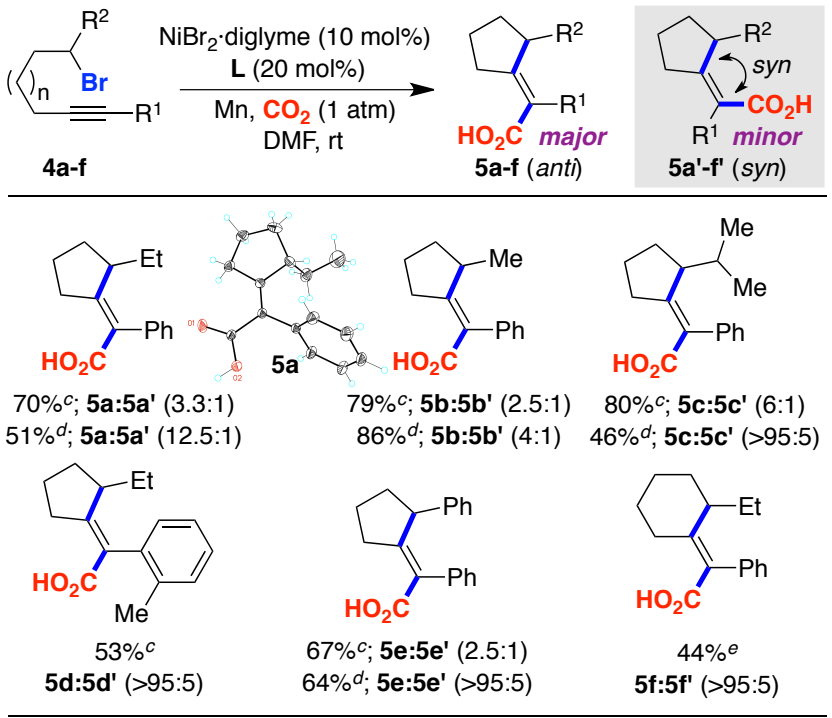

${ }^{a}$ As for Table 1 , entry $10 .^{b}$ Isolated yields, average of at least two independent runs. ${ }^{c}$ L6 was used as ligand. ${ }^{d} \mathbf{L 5}$ was used as ligand. ${ }^{e} \mathbf{L} \mathbf{4}$ was used as ligand.

On the basis of these results, we wondered whether the observed anti-selectivity switch for $\mathbf{5 a}$ could be applied to other substrate combinations. As shown in Table 3, this was indeed the case and a host of differently substituted secondary alkyl bromides could be coupled in high yields and anti-selectivities. ${ }^{21}$ Notably, six-membered carbocyclic skeletons could also be accommodated, albeit in lower yields (5f). A simple comparison of $\mathbf{5 a} v s$ $\mathbf{5 b}$ and $\mathbf{5 c}$ clearly evidences that the anti-selectivity is favored with bulkier substituents on the side chain. A similar effect was found with ortho-substituted aromatic motifs ( $\mathbf{5 d}$ vs 5a). Less counterintuitive was the observation that the ligand backbone exerted a profound influence on the selectivity pattern, with $\mathbf{L 5}$ or $\mathbf{L} \mathbf{4}$ providing the best anti/syn selectivities, thus showing the subtleties of our system. ${ }^{20}$ At present, we believe that the antiselectivity switch in secondary alkyl bromides might be attributed to the intermediacy of vinyl radical species that undergo rapid isomerization prior recombination with $\mathrm{Ni}(\mathrm{I}) \mathrm{BrLn}$ species. ${ }^{22-25}$ Taken together, the data shown in Tables 2-3 illustrate the prospective impact of our Ni-catalyzed reductive cyclization/carboxylation event from simple building blocks by promoting a distal $\mathrm{CO}_{2}$ fixation while controlling the syn/anti-selectivity pattern of the cyclization event.

\section{Scheme 3. Mechanistic Experiments.}




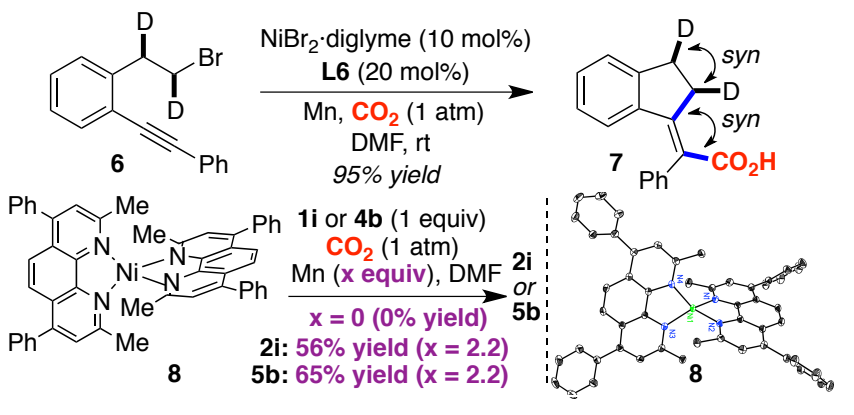

Although a detailed picture requires further studies, we decided to shed light on the mechanism by studying the stereochemical course of 6 (Scheme 3, top). As shown, careful ${ }^{1} \mathrm{H}-\mathrm{NMR}$ spectroscopical analysis revealed that the reaction exclusively afforded $7,{ }^{15}$ an observation that is consistent with a scenario consisting of an initial oxidative addition with inversion of configuration. ${ }^{26,27} \mathrm{Next}$, we turned our attention to explore the reactivity of airsensitive $\mathbf{8}$, easily accessible by simply reacting $\mathrm{Ni}(\mathrm{COD})_{2}$ with $\mathbf{L 6}$ in THF (Scheme 3 , bottom) ${ }^{28} \mathrm{Im}$ portantly, while no reaction took place upon exposure of $\mathbf{8}$ with either $1 \mathbf{i}$ or $\mathbf{4 b}$ in the absence of $\mathrm{Mn}$, the targeted cyclization/carboxylation (2i or $\mathbf{4 i}$ ) was cleanly produced in the presence of reducing agent. Although premature, we believe these experiments tacitly suggest that the carboxylation event does not occur from in situ generated $\mathrm{Ni}(\mathrm{II})$ species, but rather from putative $\mathrm{Ni}(\mathrm{I})$ reaction intermediates. ${ }^{22,29}$

In conclusion, we have developed a mild, robust and user-friendly Ni-catalyzed cascade reductive cyclization/carboxylation using $\mathrm{CO}_{2}$ at atmospheric pressure in which the selectivity pattern is dictated by an appropriate substrate and/or ligand selection. Further investigations into related processes as well as the development of an asymmetric version are currently underway.

\section{ASSOCIATED CONTENT}

Supporting Information. Experimental procedures and spectral data. This material is available free of charge via the Internet at http://pubs.acs.org.

\section{AUTHOR INFORMATION}

\section{Corresponding Author}

*rmartinromo@iciq.es

\section{Author contributions}

* These authors contributed equally to this work

\section{Funding Sources}

No competing financial interests have been declared.

\section{ACKNOWLEDGMENT}

We thank ICIQ, the European Research Council (ERC277883), MINECO (CTQ2012-34054 \& Severo Ochoa Excellence Accreditation 2014-2018, SEV-2013-0319) and
Cellex Foundation for support. Johnson Matthey, Umicore and Nippon Chemical Industrial are acknowledged for a gift of metal \& ligand sources. Y. L. thanks COFUND for a fellowship. We thank Dr. J. Cornella for preparing 8 and Eduardo Escudero for all X-Ray crystallographic data.

\section{REFERENCES}

(1) For selected reviews: (a) Moragas, T.; Correa, A.; Martin, R. Chem. Eur. J. 2014, 20, 8242. (b) Tasker, S. Z.; Standley, E. A.; Jamison, T. F. Nature 2014, 509, 299. (c) Everson, D. A.; Weix, D. J. J. Org. Chem. 2014, 79, 4793. (d) Knappe, C. E. I.; Grupe, S.; Gärtner, D.; Corpet, M.; Gosmini, C.; Jacobi von Wangelin, A. Chem. Eur. J. 2014, 20, 6828. (e) Montgomery, J. Organonickel Chemistry. In Organometallics in Synthesis; Lipshutz, B. H., Ed.; John Wiley \& Sons, Inc.; Hoboken, 2013; pp 319-428

(2) For cascade reductive coupling reactions of aryl electrophiles: Durandetti, M.; Hardou, L.; Lhermet, R.; Rouen, M.; Maddaluno, J. Chem. Eur. J. 2011, 17, 12773.

(3) For an elegant isolated example of a cyclization of alkyl halides followed by C-C bond-formation: Zhao, C.; Jia, X.; Wang, X.; Gong, H. J. Am. Chem. Soc. 2014, 136, 17645.

(4) (a) Moragas, T.; Cornella, J.; Martin, R. J. Am. Chem. Soc. 2014, 136, 17702. (b) Liu, Y.; Cornella, J.; Martin, R. J. Am Chem. Soc. 2014, 136, 11212. (c) Correa, A.; Leon, T.; Martin, R. J. Am. Chem. Soc. 2014, 136, 1062. (d) Leon, T.; Correa, A.; Martin, R. J. Am. Chem. Soc. 2013, 135, 1221. (e) Correa, A.; Martin, R. J. Am. Chem. Soc. 2009, 131, 15974.

(5) (a) Nogi, K.; Fujihara, T.; Terao, J.; Tsuji, Y. Chem. Commun. 2014, 50, 13052. (b) Tran-Vu, H.; Daugulis, O. ACS Catal. 2013, 3, 2417. (c) Fujihara, T.; Nogi, K.; Xu, T.; Terao, J.; Tsuji, Y.J. Am. Chem. Soc. 2012, 134, 9106.

(6) For selected reviews: (a) Zhang, L.; Hou, Z. Chem. Sci. 2013, 4, 3395. (b) Tsuji, Y.; Fujihara, T. Chem. Commun. 2012, 48, 9956. (c) Cokoja, M.; Bruckmeier, C.; Rieger, B.; Herrmann, W. A.; Kuhn, F. E. Angew. Chem., Int. Ed. 2011, 50, 8510. (d) Huang, K.; Sun, C.-L.; Shi, Z.-J. Chem. Soc. Rev. 2011, 40, 2435. (e) Martin, R.; Kleij, A. W. ChemSusChem 2011, 4, 1259. (f) Sakakura, T.; Choi, J. C.; Yasuda, H. Chem. Rev. 2007, 107, 2365.

(7) For selected examples: (a) Correa, A.; Martin, R. Angew. Chem. Int. Ed. 2009, 48, 6201. (b) Ukai, K.; Aoki, M.; Takaya, J.; Iwasawa, N. J. Am. Chem. Soc. 2006, 128, 8706. (c) Takaya, J.; Tadami, S.; Ukai, K.; Iwasawa, N. Org. Lett. 2008, 10, 2697. (d) Ohishi, T.; Nishiura, M.; Hou, Z. Angew. Chem., Int. Ed. 2008, 47, 5792. (d) Yeung, C. S.; Dong, V. M. J. Am. Chem. Soc. 2008, 130, 7826.

(8) (a) Patai, S. The Chemistry of Acid Derivatives; Wiley: New York, 1992. (b) Goossen, L. J.; Rodríguez, N.; Goossen, K. Angew. Chem. Int. Ed. 2008, 47, 3100. (c) Maag, H. Prodrugs of Carboxylic Acids; Springer: New Yorl, 2007.

(9) For an excellent review on tandem catalysis: Fogg, D. E.; dos Santos, E. N. Coord. Chem. Rev. 2004, 248, 2365.

(10) (a) Kambe, N.; Iwasaki, T.; Terao, J. Chem. Soc. Rev. 2011 , 40, 4937. (b) Jana, R.; Pathak, T. P.; Sigman, M. S. Chem. Rev. 2011, 111, 1417. (c) Rudolph, A.; Lautens, M. Angew. Chem., Int. Ed. 2009, 48, 2656.

(11) For selected catalytic cyclization techniques of unactivated alkyl halides using nucleophile/electrophile regimes, see: (a) Venning, A. R. O.; Bohan, P. T.; Alexanian, E. J. J. Am. Chem. Soc. 2015, 137, 3731. (b) Cong, H.; Fu, G. C. J. Am. Chem. Soc. 2014, 136, 3788. (c) Fruchey, E. R.; Monks, B. M.; Patterson, A. M.; Cook, S. P. Org. Lett. 2013, 15, 4362. (d) Monks, B. M.; Cook, S. P. J. Am. Chem. Soc. 2012, 134, 15297. (e) Bloome, K. S.; McMahen, R. L.; Alexanian, E. J. 
J. Am. Chem. Soc. 2011, 133, 20146. (f) Kim, H.; Lee, C. Org. Lett. 2011, 13, 2050. (g) Firmansjah, L.; Fu, G. C. J. Am. Chem. Soc. 2007, 129, 11340.

(12) Yoder, R. A.; Johnston, J. N. Chem. Rev. 2005, 105, 4730.

(13) Flynn, A. B.; Ogilvie, W. W. Chem. Rev. 2007, 107, 4698.

(14) For selected cyclization of well-defined alkyl organometallics with alkyne side-chains: (a) Kambe, N.; Moriwaki, Y.; Fujii, Y.; Iwasaki, T.; Terao, J. Org. Lett. 2011, 13, 4656. (b) Rao, S. A.; Knochel, P. J. Am. Chem. Soc. 1991, 113, 5735. (c) Bailey, W. F.; Ovaska, T. V.; Leipert, T. K.; Tetrahedron Lett. 1989, 30, 3901. (d) Bailey, W. F.; Ovaska, T. V. J. Am. Chem. Soc. 1983, 115, 3080. (e) Richey, H. G.; Rothman, A. M. Tetrahedron Lett. 1968, 12, 1457.

(15) See Supporting Information for details.

(16) Homodimerization accounts for the mass balance.

(17) (a) Galan, B. R.; Rovis, T. Angew. Chem. Int. Ed. 2009, 48, 2830. (b) Gandon, V.; Aubert, C.; Malacria, M. Chem. Commun. 2006, 2209. (c) Chopade, P. R.; Louie, J. Adv. Synth. Catal. 2006, 348, 2307.

(18) (a) Hartwig, J. F. Organotransition metal chemistry: from bonding to catalysis; Murdzek, J., Eds.; University Science Books: Mill Valley, CA 2010; p 379. (b) Marek, I.; Minko, Y. In Metal-catalyzed cross-coupling reactions and more, 2014; chapter 10, pp 763-874.

(19) For selected anti-carbometalation techniques using stoichiometric organometallic species: (a) Fressigné, C.; Girard, A. -L.; Durandetti, M.; Maddaluno, J. Angew. Chem. Int. Ed. 2008, 47, 891. (b) Simaan, S.; Marek, I. Org. Lett. 2007, 9, 2569. (c) Ma, S.; Negishi, E. -I. J. Org. Chem. 1997, 62, 784. (d) Lu, Z.; Ma, S. J. Org. Chem. 2006, 71, 2655.

(20) At present, the intermediacy of "redox-noninnocent ligands" cannot be ruled out, see: Hu, X. Chem. Sci. 2011, 2, 1867

(21) Unfortunately, the utilization of tertiary alkyl halides resulted in recovered starting material or reduced products.

(22) For the intermediacy of Ni species in odd oxidation states, see: (a) Laskowski, C. A.; Bungum, D. J.; Baldwin, S. M.; Del Ciello, S. A.; Iluc, V. M.; Hillhouse, G. L. J. Am. Chem. Soc. 2013, 135, 18272. (b) Breitenfeld, J.; Ruiz, J.; Wodrich, M. D.; Hu, X. J. Am. Chem. Soc. 2013, 135, 12004. (c) Biswas, S.; Weix, D. J. J. Am. Chem. Soc. 2013, 135, 16192. (d) Schley, N. D.; Fu, G. C.J. Am. Chem. Soc. 2014, 136, 16588. (e) Jones, G. D.; Martin, J. L.; McFarland, C.; Allen, O. R.; Hall, R. E.; Haley, A. D.; Brandon, R. J.; Konovalova, T.; Desrochers, P. J.; Pulay, P.; Vicic, D. A. J. Am. Chem. Soc. 2006, 128, 13175. (f) Ciszewski, J. T.; Mikhaylov, D. Y.; Holin, K. V.; Kadirov, M. K.; Budnikova, Y. H.; Sinyashin, O.; Vicic, D. A. Inorg. Chem. 2011, 50, 8630.

(23) (a) Galli, C.; Gentili, P.; Guarnieri, A.; Zvi, R. J. Org. Chem. 1996, 61, 8878. (b) Jenkins, P. R.; Symons, M. C. R.; Booth, S. E.; Swain, C. J. Tetrahedron Lett. 1992, 33, 3543. (c) Fukuyama, T.; Nishitani, S.; Inouye, T.; Morimoto, K.; Ryu, I. Org. Lett. 2006, 8, 1383. (d) Martinez-Grau, A.; Curran, D. P. Tetrahedron 1997, 53, 5679. (e) Ref. 10c

(24) For a mechanistic rationale, see ref. 15. The intermediacy of radical species gains credence by observing that the carboxylation of (iodo(2-methoxycyclopentylidene)methyl benzene $(Z / E=2: 1))$ produced a single carboxylic acid in $22 \%$ yield with $\mathbf{L 5}$ as the ligand $(E / Z>95: 5)$. At present, we cannot rule out an $E / Z$ isomerization of well-defined $\mathrm{Ni}(\mathrm{II})$ species, see for example: (a) Huggins, J. M.; Bergman, R. G. J. Am. Chem. Soc. 1981, 103, 3002. (b) Conn, R. S. E.; Karras, M.; Snider, B. B. Isr. J. Chem. 1984, 24, 108. (c) see ref. 2.

(25) We found that radical scavengers such as TEMPO, among others, did not inhibit the carboxylation of $\mathbf{1 b}$. Interestingly, an otherwise similar experiment employing $\mathbf{4 b}$ as substrate gave little conversion, if any, to $\mathbf{5 b} \mathbf{b} \mathbf{5} \mathbf{b}^{\prime}$. These results rein- force the notion that single-electron transfer processes come into play when dealing with secondary alkyl halides.

(26) For related isotopically-labelled studies with Pd catalysts: (a) ref. 10c. (b) Netherton, M. R.; Fu, G. C. Angew. Chem. Int. Ed. 2002, 41, 3910. (c) Stokes, B. J.; Opra, S. M.; Sigman, M. S. J. Am. Chem. Soc. 2012, 134, 11408.

(27) Such labelling pattern is opposite from previous work without pending alkynes in the side chain (ref. 4b). This observation is consistent with the ability of alkynes to act as non-innocent ancillary ligands. See ref. 11d for an example of such behavior.

(28) Powers, D. C.; Anderson, B. L.; Nocera, D. G. J. Am. Chem. Soc., 2013, 135, 18876

(29) Vinyl $\mathrm{Ni}(\mathrm{I})$ species have been proposed to be generated from vinyl $\mathrm{Ni}(\mathrm{II})$ by SET, see for example: (a) ref. 5c. (b) Duñach, E.; Esteves, A. P.; Medeiros, M. J.; Olivero, S. New J. Chem. 2005, 29, 633. (c) Fujihara, T.; Horimoto, Y.; Mizoe, T.; Sayyed, F. B.; Tani, Y.; Terao, J.; Sakaki, S.; Tsuji, Y. Org. Lett. 2014, 16, 4960. (d) Nadal, M. L.; Bosch, J.; Vila, J. M.; Klein, G.; Ricart, S.; Moretó, J. M. J. Am. Chem. Soc. 2005, 127, 10476. 


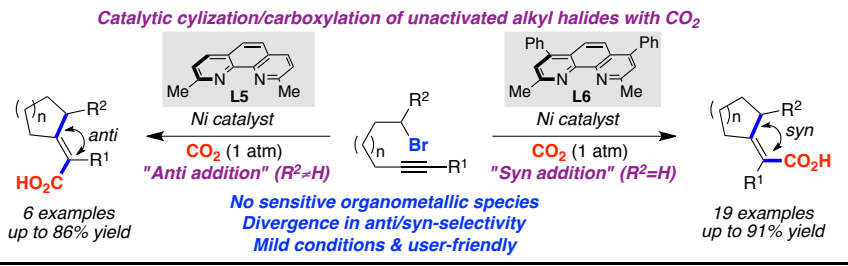

